TRANSACTIONS OF THE

AMERICAN MATHEMATICAL SOCIETY

Volume 354, Number 12, Pages 4831-4845

S 0002-9947(02)03069-6

Article electronically published on August 1, 2002

\title{
AUTOMORPHISMS OF FINITE ORDER ON GORENSTEIN DEL PEZZO SURFACES
}

\author{
D.-Q. ZHANG
}

\begin{abstract}
In this paper we shall determine all actions of groups of prime order $p$ with $p \geq 5$ on Gorenstein del Pezzo (singular) surfaces $Y$ of Picard number 1. We show that every order- $p$ element in $\operatorname{Aut}(Y)(=\operatorname{Aut}(\tilde{Y}), \tilde{Y}$ being the minimal resolution of $Y$ ) is lifted from a projective transformation of $\mathbf{P}^{2}$. We also determine when $\operatorname{Aut}(Y)$ is finite in terms of $K_{Y}^{2}$, Sing $Y$ and the number of singular members in $\left|-K_{Y}\right|$. In particular, we show that either $|\operatorname{Aut}(Y)|=2^{a} 3^{b}$ for some $1 \leq a+b \leq 7$, or for every prime $p \geq 5$, there is at least one element $g_{p}$ of order $p$ in $\operatorname{Aut}(Y)$ (hence $|\operatorname{Aut}(Y)|$ is infinite).
\end{abstract}

\section{INTRODUCTION}

We work over the complex numbers field $\mathbf{C}$. In this paper we study the pair $(X, G)$ of a normal rational surface $X$ and a finite group $G$ of automorphisms on $X$.

This subject, when $X$ is smooth, was studied by S. Kantor more than one hundred years ago $[\mathrm{K}$. It was continued by Segre, Manin, Iskovskih, Gizatullin and many others [S], [M1], M2], [I], G]. See also [H1], [H2]. In [DO], the group of automorphisms of any general del Pezzo surface is described and it turns out that its discrete part is equal to the kernel of the Cremona representation on the moduli space of $n$ points in $\mathbf{P}^{2}$. Very recently, de Fernex $[\mathrm{dF}]$ constructed all the Cremona transformations of $\mathbf{P}^{2}$ of prime order, where he employed methods different from those used by Dolgachev and the author of this paper in [ZD.

In [ZD], minimal pairs $(X, G)$ with prime order $p=|G|$ were considered. In particular, using the recent Mori theory, it was shown that if the $G$-invariant sublattice of Pic $X$ has rank 1, then $p \leq 5$ unless $X=\mathbf{P}^{2}$; the short and precise classification of these pairs, modulo equivariant isomorphism, was also given there. In $[\mathrm{MM}]$ and MZ3, a more general situation was considered where $X$ may be singular or even open.

In this paper we will consider the case where $Y$ is a Gorenstein del Pezzo singular surface of Picard number 1 . So the pair $(Y, G)$ is automatically minimal in the generalized sense of [ZD]. In contrast to the smooth case in [ZD], we shall show that every prime number $p \geq 5$ is realizable as the order of some element in $\operatorname{Aut}(Y)$ for some $Y$. This actually confirms the common belief that for a family of algebraic surfaces, the automorphism group of a special member should be larger than that of a generic member; see Remark D below. See also $\mathrm{O}$ for the case of a family of K3 surfaces.

Received by the editors March 10, 2002.

2000 Mathematics Subject Classification. Primary 14J50; Secondary 14J26.

(C)2002 American Mathematical Society 
The other contrasting result is that in the smooth case there are minimal pairs $(X, \mathbf{Z} /(5))$ with $X \neq \mathbf{P}^{2}$; so the action of $\mathbf{Z} /(5)$ on $X$ is not lifted from a regular action on $\mathbf{P}^{2}$ [ZD Table 1], while in the singular case, every element of prime order $p \geq 5$ in $\operatorname{Aut}(Y)(=\operatorname{Aut}(\tilde{Y}))$ is lifted from a projective transformation of $\mathbf{P}^{2}$ (Theorem A).

Moreover, we show that for a given $Y$, the inclusion $\mathbf{Z} /\left(p_{0}\right) \subseteq \operatorname{Aut}(Y)$ for one single prime $p_{0} \geq 5$ will guarantee the inclusion $\mathbf{Z} /(p) \subseteq \operatorname{Aut}(Y)$ for every prime $p \geq 5$ (Theorem $\mathrm{C}$ ).

We begin with a definition. A normal projective surface $Y$ is a Gorenstein del Pezzo surface if $Y$ has only rational double singularities and if the anti-canonical divisor $-K_{Y}$ is ample.

As we see from the classification of higher-dimensional algebraic varieties (see $[\mathrm{KM}]$ ), a minimal (resp. canonical) model will have some terminal (resp. canonical) singularities. In the surface case, canonical singularities are just rational double singularities (or Du Val singularities, or Dynkin type ADE singularities, or rational Gorenstein singularities in other notation). Also, Gorenstein del Pezzo surfaces $Y$ appear naturally as degenerate fibres in a family of (smooth) del Pezzo surfaces (= Fano varieties of dimension 2); the minimal model conjecture (still unsolved for dimension 4 or greater) claims that every algebraic variety is birational to either a minimal model or a variety with a Fano fibration (whose singular fibres are varieties with some mild singularities and ample anti-canonical divisor). This is also the motivation for studying singular del Pezzo surfaces.

The Gorenstein del Pezzo singular surface $Y$ of Picard number 1 satisfies $1 \leq$ $K_{Y}^{2} \leq 8$ and $K_{Y}^{2} \neq 7$ (see e.g. [MZ1]). The case $K_{Y}^{2}=8$ occurs if and only if $Y$ is the quadric cone $\bar{\Sigma}_{2}$ in $\mathbf{P}^{3}$. We now state our main results.

Theorem A. Let $p \geq 5$ be a prime number. Let $Y\left(\neq \bar{\Sigma}_{2}\right)$ be a Gorenstein del Pezzo singular surface of Picard number 1 . Then we have:

(1) Modulo equivariant isomorphism, there is either none or only one or exactly $p+1$ nontrivial $\mathbf{Z} /(p)$-action $(s)$ on $Y$.

(2) Each nontrivial $\mathbf{Z} /(p)$-action on $Y$ equals, modulo equivariant isomorphism, one of those in Examples 1.1-1.9.

(3) Every order-p element $g$ in $\operatorname{Aut}(Y)(=\operatorname{Aut}(\tilde{Y}), \tilde{Y}$ being the minimal resolution of $Y$ ) is lifted from a projective transformation $\bar{g}$ of $\mathbf{P}^{2}$, i.e., there is a birational morphism $\mu: \tilde{Y} \rightarrow \mathbf{P}^{2}$ such that $\mu(g y)=\bar{g} \mu(y)$ for all $y$ in $\tilde{Y}$.

Theorem B. Let $p \geq 5$ be a prime number. Let $Y$ be a Gorenstein del Pezzo singular surface of Picard number 1 .

(1) If $K_{Y}^{2}=6$, then there are exactly $p+1$ nontrivial $\mathbf{Z} /(p)$-actions on $Y$, modulo equivariant isomorphism.

(2) If $K_{Y}^{2}=5$, then there is a unique nontrivial $\mathbf{Z} /(p)$-action on $Y$, modulo equivariant isomorphism.

(3) If $K_{Y}^{2}$ is in $\{3,4\}$, then there is either only one or exactly $p+1$ nontrivial $\mathbf{Z} /(p)$-action(s) on $Y$, modulo equivariant isomorphism.

(4) Suppose $K_{Y}^{2}=2$. Then there is a unique (resp. there is no) nontrivial $\mathbf{Z} /(p)$ action on $Y$, modulo equivariant isomorphism, if $\operatorname{Sing} Y \neq A_{7}$ (resp. $\left.\operatorname{Sing} Y=A_{7}\right)$.

(5) Suppose $K_{Y}^{2}=1$. Then there is a unique (resp. there is no) nontrivial $\mathbf{Z} /(p)$-action on $Y$, modulo equivariant isomorphism, if $\left|-K_{Y}\right|$ has exactly two (resp. more than two) singular members (see Proposition 2.5). 
Theorem C. Let $Y$ be a Gorenstein del Pezzo singular surface of Picard number 1.

(1) The following are equivalent:

(1a) $\operatorname{Aut}(Y)$ has finite order.

(1b) $|\operatorname{Aut}(Y)|=2^{a} 3^{b}$ with $1 \leq a+b \leq 7$.

(1c) Either $\operatorname{Sing} Y=A_{7}$ (and hence $K_{Y}^{2}=2$ ), or $K_{Y}^{2}=1$ and $\left|-K_{Y}\right|$ contains at least three singular members (i.e., $K_{Y}^{2}=1$ and $Y$ is different from those four in Examples 1.1-1.4).

(2) The following are equivalent:

(2a) $\operatorname{Aut}(Y)$ has infinite order.

(2b) $\operatorname{Aut}(Y)$ contains an element of prime order $p_{0}$ with $p_{0} \geq 5$.

(2c) For each prime number $p \geq 5$, there is at least one element $g_{p}$ of order $p$ in $\operatorname{Aut}(Y)$.

Remark D. We will compare Theorems B and $\mathrm{C}$ with known results for smooth $Y$.

(1) It is known that the order of $\operatorname{Aut}(Y)$ of a generic rational surface (the blowup of $\mathbf{P}^{2}$ at very generic points) with $K_{Y}^{2} \leq 5$ is a factor of 5 ! (see [DO] or [Ko. Main Theorem]).

(2) Let $Y$ be a (smooth) del Pezzo surface with $K_{Y}^{2}=3$ or 4 . It is known that there is at most one (resp. no) $\mathbf{Z} /(p)$-action on $Y$, modulo equivariant isomorphism, if $p=5$ (resp. $p \geq 7$ is prime); see [S, pp. 147-152], [H1, Theorem 1.1] and [H2, Theorem 5.3].

An interesting observation made by I. Dolgachev is that the quotient surface of the degree 6 del Pezzo surface modulo an involution, is a 4-nodal Segre cubic surface.

(3) The following can be deduced from [DO or the main Theorem in [Ko]. Let $Y$ be a generic rational surface. If $K_{Y}^{2} \leq 4$, then there is no prime order $p(p \geq 3)$ automorphism of $Y$. Suppose $K_{Y}^{2}=5$. Then there is a unique (resp. no) nontrivial $\mathbf{Z} /(p)$-action on $Y$, modulo equivariant isomorphism, if $p=5$ (resp. if $p \geq 7$ is prime). If $K_{Y}^{2}=6,7$, then for each prime $p \geq 5$, there are exactly $p+1$ of $\mathbf{Z} /(p)$-actions on $Y$, modulo equivariant isomorphism.

(4) Let $Y$ be a Gorenstein del Pezzo singular surface of Picard number 1. [Y1] or [Y2] implies that if $K_{Y}^{2} \geq 2$, then $\operatorname{Sing} Y$ determines uniquely the isomorphism class of $Y$. For $K_{Y}^{2}=1$, see [Y1], [Y2] or Proposition 2.5.

(5) We believe that a similar classification is achievable for $p=2,3$ although the list will be much longer and less elegant.

(6) The case $K_{Y}^{2}=1$ has been considered in [Z2].

\section{EXAMPLES}

We begin with a definition. A rational elliptic (smooth) surface $f: X \rightarrow \mathbf{P}^{1}$ is called extremal if $f$ is relatively minimal (i.e., $K_{X}^{2}=0$ ) and if the Mordell-Weil group $\mathrm{MW}(f)$ of all sections is torsion (cf. $\mathrm{MP}$, $[\mathrm{OS}$ ); the latter is equivalent to saying that the Picard lattice over $\mathbf{Q}$ of $X$ is generated by a single section and fibre components.

We first give Examples 1.1-1.5, where $p \geq 5$ is a prime number and $\zeta_{p}=$ $\exp (2 \pi \sqrt{-1} / p)$. We will define an action of $\langle g\rangle \cong \mathbf{Z} /(p)$ on certain extremal rational elliptic (smooth) surfaces $X$. In the Weierstrass equations for $X$ below (cf. [MP] Tables 5.1-5.3]), we use $v$ to denote the parameter of the base curve. In Examples 1.1-1.4, a different choice of section to be blown down by the map $X \rightarrow Y$ will 
result in isomorphic $Y$ because $\mathrm{MW}(f)$ acts (on $X$ and) transitively on the set of all sections. Also, except Example 1.5, the action of $\langle g\rangle$ on $X$ stabilizes all negative curves (i.e., (-2)-curves in fibres and (-1)-curves as sections); see Theorems 2.1 and 2.2 .

Example 1.1. Let $f: X \rightarrow B \cong \mathbf{P}^{1}$ be the unique rational elliptic (smooth) surface with singular fibres of type $I I$ and $I I^{*}$. Its Weierstrass equation is given here:

$$
y^{2} z=x^{3}+v^{5} z^{3} .
$$

We define an order- $p$ automorphism $g$ on $X$ as follows:

$$
g^{*}:(x, y, z ; v) \rightarrow\left(\zeta_{p}^{-5} x, y, \zeta_{p}^{-15} z ; \zeta_{p}^{6} v\right) .
$$

Then $g$ preserves the fibration $f$ and stabilizes exactly two fibres of type $I I^{*}$ at $v=0$ and of type $I I$ at $v=\infty$. The restriction $g \mid B$ has order $p$.

Clearly, $g$ stabilizes the unique section $E$ of $f$, which is given by $[x, y, z ; v]=$ $[0,1,0 ; v]$. We label the type $I I^{*}$ fibre as $\sum_{i=1}^{6} i C_{i}+4 C_{7}+2 C_{8}+3 C_{9}$ so that $E+\sum_{i=1}^{8} C_{i}$ is a linear chain and $C_{9} \cdot C_{6}=1$. Let $X \rightarrow Y_{k}(0 \leq k \leq 4)$ be the composite of the smooth blow-down of $E+\sum_{i=1}^{k} C_{i}$ and the contraction of the (-2)-curves $\sum_{i=k+2}^{9} C_{i}$. Then $Y=Y_{k}$ satisfies

(*) $Y$ is Gorenstein del Pezzo, its Picard number $\rho(Y)=1, K_{Y}^{2}=k+1$, and Sing $Y$ is one of $E_{8}, E_{7}, E_{6}, D_{5}, A_{4}$ (depending on $k$ ).

The $g$ on $X$ induces a regular action of order- $p$ on $Y_{k}$, which we denote by the same letter $g$.

Example 1.2. Let $f: X \rightarrow B \cong \mathbf{P}^{1}$ be the unique rational elliptic (smooth) surface with singular fibres of type $I I I$ and $I I I^{*}$. Its Weierstrass equation is given here:

$$
y^{2} z=x^{3}+v^{3} x z^{2} .
$$

We define an order- $p$ automorphism $g$ on $X$ as follows:

$$
g^{*}:(x, y, z ; v) \rightarrow\left(\zeta_{p}^{-3} x, y, \zeta_{p}^{-9} z ; \zeta_{p}^{4} v\right) .
$$

Then $g$ preserves the fibration $f$ and stabilizes exactly two fibres of type $I I I^{*}$ at $v=0$ and of type $I I I$ at $v=\infty$. The restriction $g \mid B$ has order $p$.

Clearly, $g$ stabilizes the only two sections $E, E^{\prime}$ of $f$, which are given by $[x, y, z ; v]$ $=[0,1,0 ; v]$ and $[x, y, z ; v]=[0,0,1 ; v]$. We label the type $I I I^{*}$ fibre as $\sum_{i=1}^{4} i C_{i}+$ $\sum_{j=5}^{8} a_{j} C_{j}$ so that $E+\sum_{i=1}^{7} C_{i}$ is a linear chain. Let $X \rightarrow Y_{k}(k=0,1,2)$ be the composite of the smooth blow-down of $E+\sum_{i=1}^{k} C_{i}$ and the contraction of the $(-2)$-curves $\sum_{i=k+2}^{8} C_{i}$ and the $(-2)$-curve in the type $I I I$ fibre not meeting $E$. Then $Y=Y_{k}$ satisfies

(*) $Y$ is Gorenstein del Pezzo, its Picard number $\rho(Y)=1, K_{Y}^{2}=k+1$, and Sing $Y$ is one of $E_{7}+A_{1}, D_{6}+A_{1}$ and $A_{5}+A_{1}$ (depending on $k$ ).

The automorphism $g$ on $X$ induces a regular action of order- $p$ on $Y_{k}$, which we denote by the same letter $g$.

Example 1.3. Let $f: X \rightarrow B \cong \mathbf{P}^{1}$ be the unique rational elliptic (smooth) surface with singular fibres of type $I V$ and $I V^{*}$. Its Weierstrass equation is given 
here:

$$
y^{2} z=x^{3}+v^{4} z^{3} .
$$

We define an order- $p$ automorphism $g$ on $X$ as follows:

$$
g^{*}:(x, y, z ; v) \rightarrow\left(\zeta_{p}^{-2} x, y, \zeta_{p}^{-6} z ; \zeta_{p}^{3} v\right) .
$$

Then $g$ preserves the fibration $f$ and stabilizes exactly two fibres of type $I V^{*}$ at $v=0$ and of type $I V$ at $v=\infty$. The restriction $g \mid B$ has order $p$.

Clearly, $g$ stabilizes the only three sections $E, E^{\prime}, E^{\prime \prime}$ of $f$, which are given by $[x, y, z ; v]=[0,1,0 ; v]$ and $[x, y, z ; v]=\left[0, \pm v^{2}, 1 ; v\right]$. We label the type $I V^{*}$ fibre as $\sum_{i=1}^{3} i C_{i}+\sum_{j=4}^{7} a_{j} C_{j}$ so that $E+\sum_{i=1}^{5} C_{i}$ is a linear chain. Let $X \rightarrow Y_{k}$ $(k=0,1)$ be the composite of the smooth blow-down of $E+\sum_{i=1}^{k} C_{i}$ and the contraction of the $(-2)$-curves $\sum_{i=k+2}^{7} C_{i}$ and the two (-2)-curves in the type $I V$ fibre not meeting $E$. Then $Y=Y_{k}$ satisfies

(*) $Y$ is Gorenstein del Pezzo, its Picard number $\rho(Y)=1, K_{Y}^{2}=k+1$, and Sing $Y$ is one of $E_{6}+A_{2}$ and $A_{5}+A_{2}$ (depending on $k$ ).

The $g$ on $X$ induces a regular action of order- $p$ on $Y_{k}$, which we denote by the same letter $g$.

Example 1.4. Let $f: X_{J} \rightarrow B \cong \mathbf{P}^{1}$ be the rational elliptic (smooth) surface with two singular fibres of type $I_{0}^{*}$ and whose $J$-invariant of a general fibre is equal to the constant $J=4 r^{3} /\left(4 r^{3}+27 s^{2}\right)$. Here $r, s$ are in $\mathbf{C}$ so that $4 r^{3}+27 s^{2} \neq 0$. Its Weierstrass equation is given as follows, where $v$ is the parameter of the base curve

$$
y^{2} z=x^{3}+r v^{2} x z^{2}+s v^{3} z^{3} .
$$

We define an order- $p$ automorphism $g$ on $X$ as follows:

$$
g^{*}:(x, y, z ; v) \rightarrow\left(\zeta_{p}^{-1} x, y, \zeta_{p}^{-3} z ; \zeta_{p}^{2} v\right) .
$$

Then $g$ preserves the fibration $f$ and stabilizes exactly the two fibres of type $I_{0}^{*}$ at $v=0$ and $v=\infty$. The restriction $g \mid B$ has order $p$.

Clearly, $g$ stabilizes the only four sections $E, E^{\prime}, E^{\prime \prime}, E^{\prime \prime \prime}$ of $f$, which are given by $[x, y, z ; v]=[0,1,0 ; v]$ and $[x, y, z ; v]=\left[x_{i} v, 0,1 ; v\right]$, where $x_{i}$ are the roots of $x^{3}+r x+s=0$. We label a type $I_{0}^{*}$ fibre as $\sum_{i=1}^{2} i C_{i}+\sum_{j=3}^{5} a_{j} C_{j}$ so that $E+\sum_{i=1}^{3} C_{i}$ is a linear chain. Let $X_{J} \rightarrow Y_{J, k}(k=0,1)$ be the composite of the smooth blow-down of $E+\sum_{i=1}^{k} C_{i}$ and the contraction of the (-2)-curves $\sum_{i=k+2}^{5} C_{i}$ and the four $(-2)$-curves in another type $I_{0}^{*}$ fibre not meeting $E$. Then $Y=Y_{J, k}$ satisfies

(*) $Y$ is Gorenstein del Pezzo, its Picard number $\rho(Y)=1, K_{Y}^{2}=k+1$, and Sing $Y$ is one of $2 D_{4}$ and $D_{4}+3 A_{1}$ (depending on $k$ ).

The $g$ on $X_{J}$ induces a regular action of order- $p$ on $Y_{J, k}$, which we denote by the same letter $g$.

In general, if $f: X \rightarrow B\left(\cong \mathbf{P}^{1}\right)$ has singular fibre type $I_{0}^{*}, I_{0}^{*}$, then $X=X_{J}$ for some $J$ [MP, Theorem 5.4]. We remark also that the isomorphism class of $Y_{J, k}$ depends (resp. does not depend) on $J$ when $k=0$ (resp. $k=1$ ); see Proposition 2.5 .

Example 1.5. Let $f: X \rightarrow B \cong \mathbf{P}^{1}$ be the unique rational elliptic (smooth) surface with singular fibres of type $I_{1}, I_{1}, I_{5}, I_{5}$. Its Weierstrass equation is

$$
y^{2} z=x^{3}+A x z^{2}+B z^{3}
$$


where $A=-3\left(1-12 v+14 v^{2}+12 v^{3}+v^{4}\right), B=2\left(1-18 v+75 v^{2}+75 v^{4}+18 v^{5}+v^{6}\right)$. The Mordell-Weil group $G=\mathrm{MW}(f) \cong \mathbf{Z} /(5)$ acts on $X$ naturally as translations (of general fibres). In particular, it acts transitively on the set of all 5 sections. The group $G$ stabilizes every fibre. So the restriction $G \mid B$ is trivial.

In Examples 1.6-1.10 below, we let $Y$ be a Gorenstein del Pezzo singular surface of Picard number 1. Let $\tilde{Y} \rightarrow Y$ be the minimal resolution and $D$ the exceptional divisor. Denote by $\# D$ the number of irreducible components of $D$. Then the Picard number $\rho(\tilde{Y})=1+\# D$. Hence $K_{Y}^{2}=K_{\tilde{Y}}^{2}=9-\# D$. For $K_{Y}^{2} \geq 2$, Sing $Y$ determines uniquely the isomorphism class of $Y$ (see Proposition 2.5); we will also use Figure 5 in [Y1] or [Y2, Ch. 4] containing all negative curves on $\widetilde{Y}$, which are either (-1)-curves or $(-2)$-curves.

Example 1.6. Let $Y$ be the unique Gorenstein del Pezzo surface of Picard number 1 and with $\operatorname{Sing} Y=A_{2}+A_{1}$. Then $K_{Y}^{2}=6$.

We first construct such a unique surface $Y$. Let $P_{1}=[0,0,1]$ and $P_{2}=[0,1,0]$. Let $\mu: \widetilde{Y} \rightarrow \mathbf{P}^{2}$ be the blow-up of $P_{1}$ and its two infinitely near points lying on the proper transform of the line $L_{P_{1} P_{2}}$, such that $\mu^{-1}\left(L_{P_{1} P_{2}}\right)=D_{1}+E+D_{3}+D_{2}$ has the dual graph

$$
(-2)--(-1)--(-2)--(-2)
$$

Here $D_{1}$ is the proper transform of $L_{P_{1} P_{2}}$. Let $\tilde{Y} \rightarrow Y$ be the contraction of $D=\sum_{i=1}^{3} D_{i}$. Then this $Y$ is the unique Gorenstein del Pezzo surface of Picard number 1 and with $\operatorname{Sing} Y=A_{2}+A_{1}$. Note that $E, D_{i}$ are the only negative curves on $\tilde{Y}([\mathbf{Y 1}, \mathrm{Y2})$. Clearly, $G:=\operatorname{Aut}(Y)=\operatorname{Aut}(\tilde{Y})$. Since $G$ clearly stabilizes all negative curves, there is an induced $G$-action on $\mathbf{P}^{2}$ such that the map $\mu$ is $G$-equivariant. Thus $G=\left\{g \in P G L_{2}(\mathbf{C}) \mid g\left(P_{1}\right)=P_{1}, g\left(L_{P_{1} P_{2}}\right)=L_{P_{1} P_{2}}\right\}$. So $G=\left\{\left(a_{i j}\right) \in P G L_{2}(\mathbf{C}) \mid a_{i j}=0(i<j)\right\}$. Since $D_{1} \cong \mathbf{P}^{1}$, each element of $G$ of finite order fixes either two or all points of $D_{1}$.

Claim 1.6.1. Let $p \geq 2$ be a prime number. Given any point $Q \in D_{1} \backslash\left\{D_{1} \cap E\right\}$, there are exactly $p+1$ different $\mathbf{Z} /(p)$-actions on $\tilde{Y}$, modulo equivariant isomorphisms, each of which fixes at least two points of $D_{1}: Q, D_{1} \cap E$; exactly one of these $p+1$ actions fixes three and hence all points of $D_{1}$.

We use the same $Q$ to denote its image on $\mathbf{P}^{2}$ and we may assume that $Q=P_{2}$ after a change of coordinates. Now $g=\left(a_{i j}\right)$ in $G$ fixes $P_{2}$ if and only if $a_{32}=$ $a_{i j}=0(i<j)$. We may assume that $a_{11}=1$ and write $g=g_{1}+h$ where $g_{1}=\operatorname{diag}\left[1, a_{22}, a_{33}\right]$ and $h=\left(h_{i j}\right)$ with $h_{i 1}=a_{i 1}$ for $i=2,3$ and all other $h_{i j}=0$. Then $h^{2}=0$. So if $g$ is of prime order $p$, then $I_{3}=g^{p}=\left(g_{1}+h\right)^{p}=g_{1}^{p}+p g_{1}^{p-1} h$. This equality implies that $g_{1}^{p}=I_{3}$ and $h=0$. So ord $(g)=p$ if and only if $\langle g\rangle=\left\langle g_{i}\right\rangle$ for some $0 \leq i \leq p$, where $g_{p}=\operatorname{diag}\left[1,1, \zeta_{p}\right]$ and $g_{b}=\operatorname{diag}\left[1, \zeta_{p}, \zeta_{p}^{b}\right](0 \leq b \leq p-1)$ with $\zeta_{p}=\exp (2 \pi \sqrt{-1} / p)$. Now the image $\mu\left(D_{1}\right)=L_{P_{1} P_{2}}=\{X=0\}$ is $g$-fixed if and only if $g=\left[1, \zeta_{p}, \zeta_{p}\right]$. The claim follows.

For an arbitrary element $g$ of prime order $p$ in $G$, we know that $g$ fixes at least two distinct points $D_{1} \cap E$ and $Q$ on $D_{1}$. Clearly, there is a projective transformation $\tau$ mapping $P_{1}, P_{2}$ to $P_{1}$, (the image of) $Q$. This $\tau$ lifts to an automorphism $\tau$ on $\widetilde{Y}$ mapping (the pre-image of) $P_{2}$ to $Q$. Now $\left\langle\tau^{-1} g \tau\right\rangle$ fixes $P_{1}$ and $P_{2}$ and hence equals one of the $\left\langle g_{i}\right\rangle$ above for some $i$. So modulo equivariant isomorphism, $\left\langle g_{i}\right\rangle$ $(0 \leq i \leq p)$ are the only nontrivial actions of $\mathbf{Z} /(p)$ on $Y$. 
Example 1.7. Let $Y$ be the unique Gorenstein del Pezzo surface of Picard number 1 and with $\operatorname{Sing} Y=A_{3}+2 A_{1}$. Then $K_{Y}^{2}=4$.

We first construct such a unique surface $Y$. Let $P_{1}=[0,0,1], P_{2}=[0,1,0]$ and $P_{3}=[1,0,0]$. Let $\mu: \widetilde{Y} \rightarrow \mathbf{P}^{2}$ be the blow-up of $P_{1}, P_{2}$ and three infinitely near points of them such that $\mu^{-1}\left(L_{P_{1} P_{2}}+L_{P_{1} P_{3}}\right)=D_{1}+E_{1}+D_{4}+D_{3}+D_{2}+E_{2}+D_{5}$ has the dual graph

$$
(-2)--(-1)--(-2)--(-2)--(-2)--(-1)--(-2) .
$$

Here $D_{1}, D_{2}$ are the proper transforms of $L_{P_{1} P_{3}}, L_{P_{1} P_{2}}$. Let $\tilde{Y} \rightarrow Y$ be the contraction of $D=\sum_{i=1}^{5} D_{i}$. Then this $Y$ is the unique Gorenstein del Pezzo surface of Picard number 1 and with $\operatorname{Sing} Y=A_{3}+2 A_{1}$. Note that $E_{i}, D_{j}$ are the only negative curves on $\widetilde{Y}(\overline{\mathrm{Y} 1}, \mathrm{Y} 2)$. Clearly, $G:=\operatorname{Aut}(Y)=\operatorname{Aut}(\widetilde{Y})$. Let $H=\{h \in$ $\left.G \mid h\left(D_{1}\right)=D_{1}\right\}$. Then $H$ stabilizes every negative curve. As in Example 1.6, $\mu$ is $H$-equivariant and $H=\left\{h \in P G L_{2}(\mathbf{C}) \mid h\left(P_{i}\right)=P_{i}(i=1,2), h\left(L_{P_{1} P_{3}}\right)=L_{P_{1} P_{3}}\right\}$. So $H=\left\{\left(a_{i j}\right) \in P G L_{2}(\mathbf{C}) \mid a_{i j}=0\right.$ if $i \neq j$ and $\left.(i, j) \neq(3,1)\right\}$.

Claim 1.7.1. $G \cong H \rtimes \mathbf{Z} /(2)$.

Indeed, if $\sigma$ is in $G$ but not in $H$, then $\sigma$ switches $D_{1}$ and $D_{5}$ and $\sigma^{2}$ is in $H$. On the other hand, the blow-down $\widetilde{Y} \rightarrow \mathbf{P}^{1} \times \mathbf{P}^{1}$ of $E_{1}+D_{4}$ and $E_{2}+D_{2}$ to points $(0,0),(\infty, 0)$ is $G$-equivariant and the automorphism $(x, y) \mapsto(1 / x, y)$ downstairs lifts to an involution $\sigma$ in $G$ switching $D_{1}$ and $D_{5}$.

By the claim above, every prime order $p(p \geq 3)$ element $g$ of $G$ is contained in $H$. As in Example 1.6, $\langle g\rangle=\left\langle\operatorname{diag}\left[1,1, \zeta_{p}\right]\right\rangle$ or $\langle g\rangle=\left\langle\operatorname{diag}\left[1, \zeta_{p}, \zeta_{p}^{b}\right]\right\rangle(0 \leq b \leq p-1)$.

Example 1.8. Let $Y$ be the unique Gorenstein del Pezzo surface of Picard number 1 and with Sing $Y=3 A_{2}$. Then $K_{Y}^{2}=3$.

We first construct such a unique surface $Y$. Let $P_{1}=[0,0,1], P_{2}=[0,1,0]$ and $P_{3}=[1,0,0]$. Let $\mu: \widetilde{Y} \rightarrow \mathbf{P}^{2}$ be the blow-up of $P_{1}, P_{2}, P_{3}$ and three infinitely near points of them such that $\mu^{-1}\left(\sum_{i<j} L_{P_{i} P_{j}}\right)=E_{1}+D_{1}+D_{2}+E_{2}+D_{3}+D_{4}+E_{3}+$ $D_{5}+D_{6}$ is a simple loop (with $E_{1} \cdot D_{6}=1$ and) with $E_{i}^{2}=-1$ and $D_{j}^{2}=-2$. Here $D_{1}, D_{3}, D_{5}$ are the proper transforms of $L_{P_{i} P_{j}}$ with $(i, j)=(1,2),(1,3),(2,3)$. Let $\tilde{Y} \rightarrow Y$ be the contraction of $D=\sum_{i=1}^{6} D_{i}$. Then this $Y$ is the unique Gorenstein del Pezzo surface of Picard number 1 and with $\operatorname{Sing} Y=3 A_{2}$. Note that $E_{i}, D_{j}$ are the only negative curves on $\tilde{Y}(\overline{\mathrm{Y} 1},[\mathrm{Y} 2])$. Clearly, $G:=\operatorname{Aut}(Y)=\operatorname{Aut}(\tilde{Y})$. Let $H=\left\{h \in G \mid h\left(E_{i}\right)=E_{i}(i=1,2,3)\right\}$. Then $H$ stabilizes every negative curve. As in Example 1.6, the $\mu$ is $H$-equivariant and $H=\left\{h \in P G L_{2}(\mathbf{C}) \mid h\left(P_{i}\right)=P_{i}(i=\right.$ $1,2,3)\}$. So $H=\left\{\operatorname{diag}[a, b, c] \in P G L_{2}(\mathbf{C})\right\}$.

Claim 1.8.1. $G \cong H \rtimes S_{3}$.

Indeed, if $\sigma$ is in $G$ but not in $H$, then $\sigma$ permutes the $E_{i}$ 's and hence $G / H \leq S_{3}$. On the other hand, consider the blow-down $\widetilde{Y} \rightarrow \mathbf{P}^{1} \times \mathbf{P}^{1}$ of $E_{1}+D_{6}, E_{3}$ and $E_{2}+D_{3}$ to points $(0,0),(\infty, 0)$ and $(\infty, \infty)$. Then the involution $\sigma:(x, y) \mapsto(1 / y, 1 / x)$ downstairs lifts to an involution $\sigma$ in $G$ switching $E_{1}$ and $E_{2}$. Similarly, we can find an involution $\sigma_{i j}$ in $G$ switching $E_{i}$ and $E_{j}$. This proves the claim.

By the claim above, every prime order $p(p \geq 5)$ element $g$ of $G$ is contained in $H$. As in Example 1.6, $\langle g\rangle=\left\langle\operatorname{diag}\left[1,1, \zeta_{p}\right]\right\rangle$ or $\langle\bar{g}\rangle=\left\langle\operatorname{diag}\left[1, \zeta_{p}, \zeta_{p}^{b}\right]\right\rangle(0 \leq b \leq p-1)$.

Example 1.9. Let $Y$ be the unique Gorenstein del Pezzo surface of Picard number 1 and with Sing $Y=2 A_{3}+A_{1}$. Then $K_{Y}^{2}=2$. 
We first construct such a unique surface $Y$. Let $P_{1}=[0,0,1], P_{2}=[0,1,0]$, $P_{3}=[1,0,0]$ and $P_{4}=[1,1,0]$. Let $\mu: \widetilde{Y} \rightarrow \mathbf{P}^{2}$ be the blow-up of $P_{1}, P_{2}, P_{3}$ and four infinitely near points of them such that $\mu^{-1}\left(\sum_{i<j} L_{P_{i} P_{j}}\right)=L+C$ with $L=E_{1}+D_{1}+D_{2}+D_{3}+E_{4}+D_{6}+D_{5}+D_{4}$ and $C=E_{2}+D_{7}+E_{3}$. Here $E_{i}^{2}=-1$ and $D_{j}^{2}=-2$. This $L$ is a simple loop (with $E_{1} \cdot D_{4}=1$ ) and $C$ is a linear chain such that $L . C=2$ and $L$ meets $C$ at the two points $E_{2} \cap D_{2}$ and $E_{3} \cap D_{5}$. Also, $D_{1}, D_{5}, D_{6}, E_{2}$ are the proper transforms of $L_{P_{i} P_{j}}$ with $(i, j)=$ $(1,2),(2,3),(1,4),(1,3)$. Let $\widetilde{Y} \rightarrow Y$ be the contraction of $D=\sum_{i=1}^{7} D_{i}$. Then this $Y$ is the unique Gorenstein del Pezzo surface of Picard number 1 and with Sing $Y=2 A_{3}+A_{1}$. Note that $E_{i}, D_{j}$ are the only negative curves on $\tilde{Y}$ ([Y1], Y2]). Clearly, $G:=\operatorname{Aut}(Y)=\operatorname{Aut}(\tilde{Y})$. Let $H=\left\{h \in G \mid h\left(E_{i}\right)=E_{i}(1 \leq i \leq 4)\right\}$. Then $H$ stabilizes every negative curve. As in Example 1.6, $\mu$ is $H$-equivariant and $H=\left\{h \in P G L_{2}(\mathbf{C}) \mid h\left(P_{i}\right)=P_{i}(1 \leq i \leq 4)\right\}$. So $H=\left\{\operatorname{diag}[1,1, c] \in P G L_{2}(\mathbf{C})\right\}$.

Claim 1.9.1. $G \cong H \rtimes(\mathbf{Z} /(2))^{\oplus 2}$.

Indeed, if $\sigma$ is in $G$ but not in $H$, then $\sigma$ stabilizes both sets $\left\{E_{1}, E_{4}\right\}$ and $\left\{E_{2}, E_{3}\right\}$. Note that the blow-down $\widetilde{Y} \rightarrow \mathbf{P}^{1} \times \mathbf{P}^{1}$ of $E_{1}, E_{4}, E_{2}+D_{2}$ and $E_{3}+$ $D_{5}$ to points $(0, \infty),(\infty, 0),(0,0),(\infty, \infty)$ is $G$-equivariant. The involutions $\sigma_{1}$ : $(x, y) \mapsto(1 / y, 1 / x)$ and $\sigma_{2}:(x, y) \mapsto(y, x)$ lift to involutions $\sigma_{1}, \sigma_{2}$ in $G$ switching respectively $E_{2}$ and $E_{3}, E_{1}$ and $E_{4}$. This proves the claim.

By the claim above, every prime order $p(p \geq 3)$ element $g$ of $G$ is contained in $H$. Arguing as in Example 1.6, $\langle g\rangle=\left\langle\operatorname{diag}\left[1,1, \zeta_{p}\right]\right\rangle$.

Example 1.10. Let $Y$ be the unique Gorenstein del Pezzo surface of Picard number 1 and with $\operatorname{Sing} Y=A_{7}$. Then $K_{Y}^{2}=2$. We shall show that $\operatorname{Aut}(Y)=$ $\mathbf{Z} /(4) \times \mathbf{Z} /(2)$.

We first construct such a unique surface $Y$. Let $P_{1}=(0,0)$ and $P_{2}=(\infty, 0)$. Let $\mu: \widetilde{Y} \rightarrow \mathbf{P}^{1} \times \mathbf{P}^{1}$ be the blow-up of $P_{1}, P_{2}$ and four infinitely near points of them such that $\mu^{-1}\left(L_{P_{1}}+M+L_{P_{2}}\right)=E_{1}+D+E_{2}$ with $D=D_{1}+\cdots+D_{7}$; here $L_{P_{i}}(i=1,2)$ are the fibres $x=0$ and $x=\infty$, and $M$ is the section $y=0$. Also, $E_{i}^{2}=-1$ and $D_{j}^{2}=-2$. This $D$ is a linear chain such that $E_{i} . D=E_{i} . D_{j}=1$ with $(i, j)=(1,2),(2,6)$. Also, $D_{1}, D_{4}, D_{7}$ are the proper transforms of $L_{P_{1}}, M$, $L_{P_{2}}$. Let $\widetilde{Y} \rightarrow Y$ be the contraction of $D$. Then this $Y$ is the unique Gorenstein del Pezzo surface of Picard number 1 and with $\operatorname{Sing} Y=A_{7}$. Note that $E_{i}, D_{j}$ are the only negative curves on $\tilde{Y}(\overline{\mathrm{Y} 1}],[\mathrm{Y} 2])$.

Clearly, $G:=\operatorname{Aut}(Y)=\operatorname{Aut}(\widetilde{Y})$. Let $H=\left\{h \in G \mid h\left(E_{i}\right)=E_{i}(i=1,2)\right\}$. Then $H$ stabilizes every negative curve. Note that the restriction $H \mid D_{i}$ with $i=2$ (resp. $i=6$ ) is trivial for $H$ fixes three intersection points of $D_{i}\left(\cong \mathbf{P}^{1}\right)$ with $D_{i-1}$, $D_{i+1}$ and $E_{1}$ (resp. $E_{2}$ ). As in Example 1.6, $\mu$ is $G$-equivariant. So $H=\{h \in$ $\left.\operatorname{Aut}\left(\mathbf{P}^{1} \times \mathbf{P}^{1}\right)\left|h\left(P_{i}\right)=P_{i}(i=1,2) ; h\right| D_{j}=\operatorname{id}(j=2,6)\right\}$.

Since an element $h$ in $H$ stabilizes the curves $L_{P_{1}}=\{x=0\}$ and $M=\{y=0\}$, we have $h:(x, y) \mapsto(a x, b y)$. Following the blow-up process, we see that $h \mid D_{j}=$ id $(j=2,6)$ if and only if $a=b^{2}$ and $a^{-1}=b^{2}$. Thus $H=\left\langle h_{1}\right\rangle \cong \mathbf{Z} /(4)$, where $h_{1}=(-1, \sqrt{-1})$. Let $\sigma_{c}:(x, y) \mapsto(c / x, y)$ be an involution. Then for a unique choice of $c$, the $\sigma_{c}$ lifts to an involution on $\tilde{Y}$ switching $E_{1}$ and $E_{2}$. Clearly, $h_{1} \sigma_{c}=\sigma_{c} h_{1}$. Therefore, $G=\left\langle h_{1}\right\rangle \times\left\langle\sigma_{c}\right\rangle \cong \mathbf{Z} /(4) \times \mathbf{Z} /(2)$. 


\section{Proofs of Theorems}

We will first prove Theorems 2.1-2.3. Theorems A, B and C will follow from Theorem 2.3, the observation that $K_{Y}^{2}=9-\# D$ in Proposition 2.5, Proposition 2.5 (3) and Lemma 2.6 (4).

Theorem 2.1. Let $p \geq 5$ be a prime number. Let $f: X \rightarrow \mathbf{P}^{1}$ be an extremal rational elliptic (smooth) surface such that $\mathbf{Z} /(p)$ acts nontrivially on $X$. Then modulo equivariant isomorphism, the $\mathbf{Z} /(p)$-action equals one of those in Examples 1.1-1.5 (4 isolated ones and a family parametrized by a parameter $J$ in $\mathbf{C}$ ). In particular, $\mathbf{Z} /(q) \subseteq \operatorname{Aut}(X)$ for every prime $q \geq 5$, unless $p=5$ and $X$ is given in Example 1.5.

Theorem 2.2. Let $p \geq 5$ be a prime number. Let $f: X \rightarrow \mathbf{P}^{1}$ be an extremal rational elliptic (smooth) surface. Then a nontrivial action (if it exists) of $\mathbf{Z} /(p)$ on $X$ is unique modulo equivariant isomorphism. Also, the action stabilizes all negative curves on $X$, unless $p=5$ and $X$ is given in Example 1.5.

Theorem 2.3. Let $p \geq 5$ be a prime number. Let $Y$ be a Gorenstein del Pezzo singular surface of Picard number 1.

(1) $Y$ admits a nontrivial $\mathbf{Z} /(p)$-action if and only if either $K_{Y}^{2} \geq 2$ and $\operatorname{Sing} Y \neq$ $A_{7}$, or $K_{Y}^{2}=1$ and $\left|-K_{Y}\right|$ has exactly two singular members (i.e., $K_{Y}^{2}=1$ and $Y$ equals one of those four in Examples 1.1-1.4) whence $\operatorname{Sing} Y$ is one of the following (see Proposition 2.5):

$$
E_{8}, E_{7}+A_{1}, E_{6}+A_{2}, 2 D_{4}
$$

(2) If $K_{Y}^{2}=1$, then there is at most one nontrivial $\mathbf{Z} /(p)$-action on $Y$, modulo equivariant isomorphism.

(3) If $\operatorname{Sing} Y$ is either $A_{2}+A_{1}$, or $A_{3}+2 A_{1}$ or $3 A_{2}$, then there are exactly $p+1$ different nontrivial $\mathbf{Z} /(p)$-actions on $Y$, modulo equivariant isomorphism (see Examples 1.6-1.8).

(4) If $\operatorname{Sing} Y=A_{7}$, then $\operatorname{Aut}(Y) \cong \mathbf{Z} /(4) \oplus \mathbf{Z} /(2)$ (see Example 1.10).

(5) If $K_{Y}^{2} \geq 2, Y \neq \bar{\Sigma}_{2}$ and $\operatorname{Sing} Y$ is none of those four in (3) and (4), then there is a unique nontrivial $\mathbf{Z} /(p)$-action on $Y$, modulo equivariant isomorphism.

We need some preparations.

Lemma 2.4. Let $f: X \rightarrow B\left(\cong \mathbf{P}^{1}\right)$ be an extremal rational elliptic (smooth) surface such that there are at least three singular fibres. Then either $|\operatorname{Aut}(X)|=2^{a} 5$ with $1 \leq a \leq 3$ or $|\operatorname{Aut}(X)|=2^{a} 3^{b}$ with $1 \leq a+b \leq 7$. In the first case, every element of order 5 acts transitively on the set of the 5 sections of $f$.

Proof. Let $F$ be the generic fibre of $f$ over the function field $\mathbf{C}(B)$. We have the exact sequence (see G, p. 128])

$$
(1) \rightarrow \operatorname{Aut}(F) \rightarrow \operatorname{Aut}(X) \rightarrow \operatorname{Aut}(B) .
$$

Let $F_{1}=f^{-1}\left(t_{1}\right), \ldots, F_{r}=f^{-1}\left(t_{r}\right)$ be all singular fibres of $f$. Then $H=$ $\operatorname{Im}(\operatorname{Aut}(X) \rightarrow \operatorname{Aut}(B))$ acts on the set $\left\{t_{1}, \ldots, t_{r}\right\}$. The natural map $H \rightarrow S_{r}$ is injective for $r \geq 3$ and $B \cong \mathbf{P}^{1}$. If we divide $\left\{t_{1}, \ldots, t_{r}\right\}$ into a disjoint union of subsets of cardinality $n_{k}$ with $\sum_{k} n_{k}=r$, such that fibres over points in the same subset are of the same type, then $H$ stabilizes each subset and hence $|H|$ divides $\Pi_{k}\left(n_{k}\right)$ !. 
On the other hand, if we let MW $(f)$ be the Mordell-Weil group of all sections of $f$ and $\operatorname{Aut}(F)_{0}$ be the subgroup of $\operatorname{Aut}(F)$ fixing the zero element (coming from a predesignated zero section of $f)$, then $\operatorname{Aut}(F)=\operatorname{MW}(f) \rtimes \operatorname{Aut}(F)_{0}$. So we conclude that $|\operatorname{Aut}(X)|=\left|\operatorname{Aut}(F)_{0}\right||\mathrm{MW}(f)||H|$. All such $f$ are given in $[\mathrm{MP}$ Theorem 4.1] (see also [ibid, Theorem 5.4]). By [ibid, Tables 5.1-5.3], the $J$-function $J(F)$ is not a constant. So $\left|\operatorname{Aut}(F)_{0}\right|=2$. Now the lemma follows from the classification of singular fibre types and MW $(f)$ in [ibid, Theorem 4.1] and the above observation that $|H|$ divides $\Pi_{k}\left(n_{k}\right)$ !.

Proposition 2.5. Let $Y\left(\neq \bar{\Sigma}_{2}\right)$ be a Gorenstein del Pezzo (singular) surface of Picard number 1.

(1) Sing $Y$ is one of the following (26 of them):

$$
E_{8}, E_{7}+A_{1}, E_{6}+A_{2}, 2 D_{4}, D_{8}, D_{5}+A_{3}, D_{6}+2 A_{1}, A_{8}, A_{7}+A_{1},
$$$$
2 A_{4}, A_{5}+A_{2}+A_{1}, 2 A_{3}+2 A_{1}, 4 A_{2}, E_{7}, D_{6}+A_{1}, A_{7}, A_{5}+A_{2}, D_{4}+3 A_{1} \text {, }
$$

$$
2 A_{3}+A_{1}, 3 A_{2}, A_{5}+A_{1}, E_{6}, A_{3}+2 A_{1}, D_{5}, A_{4}, A_{2}+A_{1} \text {. }
$$

(2) Sing $Y$ determines uniquely the isomorphism class of $Y$, and $\left|-K_{Y}\right|$ has at least three singular members, if $\operatorname{Sing} Y$ is not $E_{8}, E_{7}+A_{1}, E_{6}+A_{2}$ or $2 D_{4}$. If $D$ is one of $E_{8}, E_{7}+A_{1}$ and $E_{6}+A_{2}$, then there are exactly two isomorphism classes of $Y=Y_{D}(i)$ with $\operatorname{Sing} Y=D ; Y=Y_{D}(1)$ is given in Examples $1.1-1.3 ;$ the $\left|-K_{Y}\right|$ with $Y=Y_{D}(1)$ (resp. $Y=Y_{D}(2)$ ) has exactly two (resp. at least three) singular members. If Sing $Y=2 D_{4}$, then $Y$ is isomorphic to one of those $Y_{J, 0}$ in Example 1.4 parametrized by $J$ in $\mathbf{C}$, and $\left|-K_{Y}\right|$ has exactly two singular members.

(3) Suppose that $K_{Y}^{2}=1$ and $\left|-K_{Y}\right|$ contains at least three singular members. Then $|\operatorname{Aut}(Y)|=2^{a} 3^{b}$ with $1 \leq a+b \leq 7$.

For the $Y$ in the proposition above, we let $\tilde{Y} \rightarrow Y$ be the minimal resolution and $D$ the exceptional divisor. Clearly, $\operatorname{Aut}(Y)=\operatorname{Aut}(\tilde{Y})$. Also, the Picard number $\rho(\widetilde{Y})=1+\# D$ and $K_{Y}^{2}=K_{\widetilde{Y}}^{2}=9-\# D$, where $\# D$ is the number of irreducible components of $D$.

Proof of Proposition 2.5. (1) is well known (see, e.g., [MZ1]). (2) is proved in [Y1], [Y2]. We state the idea of the proof of (2) for the case $K_{Y}^{2}=1$. Let $X \rightarrow \widetilde{Y}$ be the blow-up of the unique point Bs $\left|-K_{\tilde{Y}}\right|$ with $E$ the exceptional curve (see the proof of Theorem 2.3 below $)$. Then $f=\Phi_{\left|-K_{X}\right|}: X \rightarrow B\left(\cong \mathbf{P}^{1}\right)$ is an extremal rational elliptic surface. These $X, \mathrm{MW}(f)$ and isomorphism classes are classified in [MP, Theorems 4.1 and 5.4]. The composition map $X \mapsto Y$ defines a one-to-one correspondence between the set of isomorphism classes of extremal rational elliptic (smooth) surfaces and the set of Gorenstein del Pezzo surfaces of Picard number 1 and degree 1 ; the singular members of $\left|-K_{Y}\right|$ are exactly the images of singular fibres of $f$.

Assertion (3) follows from Lemma 2.4 and the observation that $\operatorname{Aut}(Y)=\{g \in$ $\operatorname{Aut}(X) \mid g(E)=E)\} \supseteq\langle\sigma\rangle$; here $E$ is a section of $f$ and $\sigma$ acts as - id on the generic fibre (fixing the zero section, which is chosen to be $E$ ).

Lemma 2.6. Let $Y$ be as in the proposition above. Let $g$ be an element in $\operatorname{Aut}(Y)$ $=\operatorname{Aut}(\tilde{Y})$ of prime order $p(p \geq 5)$.

(1) Every negative curve on $\widetilde{Y}$ is either a (-1)-curve or a (-2)-curve, and all negative curves are $g$-stable. If $K_{Y}^{2} \geq 2$, then there are at most four of the $(-1)$ curves on $\tilde{Y}$. 
(2) If $E$ is a $(-1)$-curve on $\tilde{Y}$, then $-K_{\tilde{Y}}=d E+\Delta$ numerically, where $d=K_{Y}^{2}$ and $\Delta$ is an effective $\mathbf{Q}$-divisor with support in $D$.

(3) There is a $(-1)$-curve $E$ on $\tilde{Y}$ such that $E . D=1$ if $\operatorname{Sing} Y$ equals one of the following:

$$
E_{7}, D_{6}+A_{1}, A_{7}, A_{5}+A_{2}, A_{5}+A_{1}, E_{6}, D_{5}, A_{4} .
$$

(4) Theorem A (3) in the Introduction is true.

Proof. The nef and bigness of $-K_{\tilde{Y}}$ implies that every negative curve is either a $(-1)$-curve or a $(-2)$-curve. When $K_{Y}^{2}=1,(1)$ is reduced to Theorem 2.2 (see the proof of Theorem 2.3). (3) and the second part of (1) follow from Figure 5 in Y1 or [Y2, Ch. 4]. In particular, when $K_{Y}^{2} \geq 2$, all (-1)-curves are $g$-stable. Also, every component of $D$ of the type as shown in Proposition 2.5 is clearly $g$-stable. So (1) follows.

For (2), we note that the Picard number $\rho(Y)=1$, whence we have numerically $-K_{Y}=b \bar{E}$ for some rational number $b$, where $\bar{E}$ is the image on $Y$ of $E$. Pulling back this relation, we get $-K_{\tilde{Y}}=b\left(E+\Delta^{\prime}\right)$, where $\Delta^{\prime}$ is an effective $\mathbf{Q}$-divisor and supported by $D$. Intersecting this with $-K_{\widetilde{Y}}$, we get $b=K_{\tilde{Y}}^{2}=K_{Y}^{2}$.

To prove (4), we need:

Claim 2.6.1. There is a birational morphism $\mu: \tilde{Y} \rightarrow \mathbf{P}^{2}$.

By (1), we have a birational morphism $\tilde{Y} \rightarrow \Sigma_{d}$ where $\Sigma_{d}$ is the Hirzebruch surface of degree $d \leq 2$. We let $M$ be the section on $\Sigma_{d}$ with $M^{2}=-d$. Since $K_{\tilde{Y}}^{2}<8$, the map $\tilde{Y} \rightarrow \Sigma_{d}$ factors through the blow-up $Z \rightarrow \Sigma_{d}$ of a point on a fibre $F$. The inverse on $Z$ of $F$ is a pair $E+F^{\prime}$ of intersecting (-1)-curves with $F^{\prime}$ the proper transform of $F$. If $d=1$, then the claim is clear. If $d=0,2$, we have a composition $\widetilde{Y} \rightarrow Z \rightarrow \Sigma_{1}$ where the latter map is the blow-down of $F^{\prime}$. The claim follows.

We continue the proof of Lemma 2.6 (4). By (1), the $\mu$-exceptional divisor is $g$-stable. So there is an induced action of $g$ on $\mathbf{P}^{2}$ so that $\mu$ is $g$-equivariant. Now Theorem A (3) follows. This proves the lemma.

Let $X$ be a smooth projective surface with an order- $n$ automorphism $g$. Let $C_{0}+C_{1}+\cdots+C_{r}$ be a linear chain of $(-2)$-curves each of which is $g$-stable. Denote by $P_{i+1}=C_{i} \cap C_{i+1}$. Note that the fixed set $C_{i}^{g}$ contains two distinct points $P_{i}, P_{i+1}$, where $P_{0} \in C_{0}$ and $P_{r+1} \in C_{r}$. Let $\zeta$ be a primitive $n$th root of 1 .

Lemma 2.7. Let $g \mid P_{i}=\left(\zeta^{a_{i}}, \zeta^{b_{i}}\right)$ be the diagonalization. Then we have $b_{i}+a_{i+1}=$ $0(\bmod n)$ and $a_{i}+b_{i}=a_{j}+b_{j}(\bmod n)$ For all $i$ and $j$.

Proof. The first equality follows from the fact that if $g$ acts as a multiple $\zeta^{e}$ at the origin of $C_{i} \cong \mathbf{P}^{1}$, then it acts as a multiple $\zeta^{-e}$ at infinity. For the second equality, we note that the restricted line bundle $\mathcal{O}\left(K_{X}\right) \mid C_{i}$ is trivial since $C_{i}$ is a (-2)-curve, and $g$ acts on it by a constant multiple $g:\left(d x_{i} \wedge d y_{i}\right)\left|C_{i} \mapsto \zeta^{a_{i}+b_{i}}\left(d x_{i} \wedge d y_{i}\right)\right| C_{i}$, where $x_{i}, y_{i}$ are defining equations of $C_{i}, C_{i+1}$ at $P_{i}$. So $\zeta^{a_{i}+b_{i}}=\zeta^{a_{i+1}+b_{i+1}}$ since $P_{i}$ and $P_{i+1}$ are on the same curve $C_{i}$. This proves the lemma.

In Lemmas 2.8 and 2.9 below, we let $f: X \rightarrow B \cong \mathbf{P}^{1}$ be an extremal rational elliptic (smooth) surface and $g$ an element in $\operatorname{Aut}(X)$ of prime order $p(p \geq 5)$. Note that each fibre is a member of $\left|-K_{X}\right|$. So the fibration is $g$-stable. We denote by $g \mid B$ the induced action of $g$ on the base curve $B$. 
Lemma 2.8. Suppose that $g \mid B=\mathrm{id}$. Then $f$ has singular fibres of types $I_{5}, I_{5}, I_{1}$, $I_{1}$ (such an $X$ is unique; see $[\mathrm{MP}), p=5$ and $\mathrm{MW}(f) \cong \mathbf{Z} /(5)$. Moreover, the action of $\langle g\rangle \cong \mathbf{Z} /(5)$ on $X$ is identical to that of $\mathrm{MW}(f)$ on $X$ as translations (on a general fibre).

Proof. If $p$ does not divide $|\mathrm{MW}(f)|$, then $g$ stabilizes each section; so $g$ fixes each section for $g \mid B=\mathrm{id}$. Thus a general fibre $F$ is an elliptic curve on which $g$ acts with a fixed point (the intersection of $F$ and a section). So ord $(g \mid F)$ divides 12 . On the other hand, $\operatorname{ord}(g \mid F)$ divides $\operatorname{ord}(g \mid X)=p \geq 5$. So $g \mid F=\mathrm{id}$. This and $g \mid B=\mathrm{id}$ imply that $g=\operatorname{id}_{X}$, a contradiction. Thus $p$ divides $|\mathrm{MW}(f)|$. Now the first part of the lemma follows from [MP , Theorems 4.1 and 5.4]. Also, $g$ acts transitively on the set of all 5 sections by the argument above.

For the second part, choose an automorphism $h$ of $X$ coming from an element (denoted also by $h$ ) of $\mathrm{MW}(f)$ such that $g^{-1} h$ acts identically on the set of the 5 sections in $\mathrm{MW}(f)$. Then $k=g^{-1} h$ acts on a general fibre $F$ with at least 5 fixed points (the intersection of $F$ with the 5 sections). Note that $\operatorname{ord}(k \mid F)$ divides 12 and if $k \mid F \neq \pm$ id, then $J(F)=0$ or 1728 . On the other hand, $J(F)$ is not a constant by [MP, Table 5.3]. So $k \mid F= \pm \mathrm{id}$. If $k \mid F=\mathrm{id}$, then $k=\mathrm{id}_{X}$ for $k \mid B=\mathrm{id}$, whence $g=h$. If $k \mid F=-\mathrm{id}$, then $g \mid F=\left(-\mathrm{id}_{F}\right) h$ has order 10, a contradiction. This proves the lemma.

Lemma 2.9. Suppose that $g \mid B \neq$ id. Then $f$ has one of the following four singular fibre types:

(i) $I I, I I^{*}$,

(ii) $I I I, I I I^{*}$,

(iii) $I V, I V^{*}$,

(iv) $I_{0}^{*}, I_{0}^{*}$.

Moreover, for each $k=1,2,3$, there is a unique $X$ of singular fibre type $(k)$ above. The surfaces with singular fibre type $(i v)$ above are parametrized by the $J$-invariant of a general fibre $F(J(F)$ is a constant).

Proof. If one singular fibre is not $g$-stable, then $f$ has $p(p \geq 5)$ copies of the same fibre, which contradicts the fact that $f$ has at most four singular fibres $\mathrm{MP}$. Theorem 4.1]. So every singular fibre is $g$-stable. If $f$ has more than two singular fibres, then $g \mid B$ fixes more than two points (over which lie the singular fibres); so $g \mid B=$ id for $B \cong \mathbf{P}^{1}$. This is a contradiction. Thus $f$ has at most two singular fibres. Now the lemma follows from [MP, Theorem 4.1].

In view of Lemmas 2.8 and 2.9, to show Theorems 2.1 and 2.2 we have only to show that for each $X$ satisfying one of the 4 cases in Lemma 2.9, there is a unique nontrivial $G=\mathbf{Z} /(p)$-action $(p \geq 5)$ on $X$ (one is given in Examples 1.1-1.4). Note that in all 4 cases, $|\mathrm{MW}(f)| \leq 4$ and hence $G$ acts trivially on the set of sections, i.e., $G$ stabilizes every section, because $p \geq 5$. This and $p \geq 5$ again imply that every component in the two singular fibres (each of which is $G$-stable) is $G$-stable and hence the central component $C_{0}$, meeting three other components $C_{1}, C_{2}, C_{3}$, of a singular fibre is $G$-fixed (pointwise). Let $P=C_{0} \cap C_{1}$ and let $h$ be a generator of $G$ such that $h \mid P$ can be diagonalized as $h \mid P=\left(1, \zeta_{p}\right)$, where $h$ acts identically along the direction of $C_{0}$ and as a multiple $\zeta_{p}=\exp (2 \pi \sqrt{-1} / p)$ along the direction of $C_{1}$. Applying Lemma 2.7, we can show that the $g$ in Examples 1.1-1.4 satisfies $g \mid P=\left(1, \zeta_{p}\right)$ so that $g^{-1} h$ acts identically along the directions of $C_{0}$ and $C_{1}$. Hence $g^{-1} h=\operatorname{id}_{X}$ and $h=g$. This shows that the nontrivial action of $\mathbf{Z} /(p)$ on $X$ is 
the same as that of $\langle g\rangle \cong \mathbf{Z} /(p)$ on $X$ in any of Examples 1.1-1.4. This proves Theorems 2.1 and 2.2 .

We can avoid the use of Lemma 2.7 by choosing a suitable power $g^{e}$ so that $g^{e} \mid P=\left(1, \zeta_{p}\right)$, whence $h=g^{e}$; so Lemma 2.7 claims that $e=1$ indeed.

Next we prove Theorem 2.3 by reducing to Theorems 2.1 and 2.2. Let $\widetilde{Y} \rightarrow Y$ and $D$ be as in Proposition 2.5. Assertion (4) is proved in Example 1.10. If $K_{Y}^{2} \geq 7$, then $K_{Y}^{2}=8$ and $Y$ equals the quadric cone $\bar{\Sigma}_{2}$ in $\mathbf{P}^{3}$ (see [MZ1], or Proposition 2.5 ); in this case, Theorem 2.3 can be checked easily in the spirit of Example 1.6. So we may assume that $\operatorname{Sing} Y \neq A_{7}$ and $K_{Y}^{2} \leq 6$.

We first consider the case $K_{Y}^{2}=1$. By the Riemann-Roch theorem and the Kawamata-Viehweg Vanishing [Ka], $[\mathrm{V}]$, the linear system $\left|-K_{\tilde{Y}}\right|$ has dimension 1 , and it has a unique base point $Q$ by [D, Proposition 2, p. 40]. Let $X \rightarrow \widetilde{Y}$ be the blow-up of $Q$ with $E$ the exceptional curve. Then the linear system $\left|-K_{X}\right|$ has dimension 1 and is base point free; so it defines an elliptic fibration $f: X \rightarrow B \cong \mathbf{P}^{1}$ with $E$ as a section. Clearly, the nontrivial action of $\mathbf{Z} /(p)$ on $Y$ induces a nontrivial action of $\mathbf{Z} /(p)$ on $X$ so that the birational morphism $X \rightarrow Y$ is $\mathbf{Z} /(p)$-equivariant. Since the Picard number $\rho(Y)=1$, we see easily that $f$ is extremal in the sense of [MP]. Now for Theorem 2.3, the case $K_{Y}^{2}=1$ follows from Theorems 2.1 and 2.2. Indeed, since $\mathbf{Z} /(p)$ stabilizes the section $E$, this $X$ is not equal to the surface in Example 1.5.

Next, we consider the case $d:=K_{Y}^{2} \geq 2$. Suppose that $\operatorname{Sing} Y$ is as in Lemma 2.6 (3). Then there is a $(-1)$-curve $E_{d}$ on $\widetilde{Y}$ such that $E_{d} \cdot D=1$. If there is an element $g$ in $\operatorname{Aut}(Y)=\operatorname{Aut}(\tilde{Y})$ of prime order $p(p \geq 5)$, then $g$ fixes at least two points on $E_{d}\left(\cong \mathbf{P}^{1}\right): E_{d} \cap D, Q_{d}$. Let $\sigma: \widetilde{Y}_{d-1} \rightarrow \widetilde{Y}$ be the blow-up of $Q_{d}$ with $E_{d-1}$ the exceptional curve. Then the anti-canonical divisor of $\widetilde{Y}_{d-1}$ equals $\sigma^{*}\left(-K_{\tilde{Y}}\right)-E_{d-1}=d E_{d}^{\prime}+(d-1) E_{d-1}+\sigma^{*}(\Delta) \geq 0$ in the notation of Lemma 2.6 (where $E_{d}^{\prime}$ is the proper transform of $E_{d}$ ), and hence it is nef and also big because its self-intersection equals $d-1 \geq 1$. So the divisor $D_{d-1}=\sigma^{*}(D)+E_{d}^{\prime}$ of $(-2)$ curves, which has zero intersection with the nef and big anti-canonical divisor of $\widetilde{Y}_{d-1}$, is contractible to rational double singularities by the map $\widetilde{Y}_{d-1} \rightarrow Y_{d-1}$. Also, $Y_{d-1}$ has Picard number 1 and ample $-K_{Y_{d-1}}$. Clearly, our $g$ in $\operatorname{Aut}(\tilde{Y})=\operatorname{Aut}(Y)$ induces an element $g$ in $\operatorname{Aut}\left(\widetilde{Y}_{d-1}\right)=\operatorname{Aut}\left(Y_{d-1}\right)$ so that $\sigma$ is $g$-equivariant.

In particular, if $d=2$, then Sing $Y_{d-1}$ is one of the four in Theorem 2.3 (1) and also $\langle g\rangle \cong \mathbf{Z} /(p)$ in $\operatorname{Aut}\left(Y_{d-1}\right)$ is unique modulo equivariant isomorphism; hence such $\langle g\rangle$ in $\operatorname{Aut}(Y)$ is also unique for any given $Y$ (see Lemma 2.6 (1)) modulo equivariant isomorphism.

For general $d \geq 2$, we have blow-ups below to reduce to the case $d=1: \widetilde{Y}_{1} \rightarrow$ $\tilde{Y}_{2} \rightarrow \cdots \rightarrow \widetilde{Y}_{d}$. Using the information on the location of the point $E_{d} \cap D$ in $D$ given in Figure 5 in [Y1] or [Y2, Ch. 4], all possible chains of singularity types Sing $Y_{1} \rightarrow \operatorname{Sing} Y_{2} \rightarrow \cdots \rightarrow \operatorname{Sing} Y_{d}$ are as follows:

$$
\begin{gathered}
E_{8} \rightarrow E_{7} \rightarrow E_{6} \rightarrow D_{5} \rightarrow A_{4}, \\
E_{7}+A_{1} \rightarrow D_{6}+A_{1} \rightarrow A_{5}+A_{1}, \\
E_{6}+A_{2} \rightarrow A_{5}+A_{2} .
\end{gathered}
$$

So Theorem 2.3 is true if $\operatorname{Sing} Y$ is one of those in Lemma 2.6 (3). 
We are left with those $Y$ with $\operatorname{Sing} Y$ equal to one of the following:

$$
D_{4}+3 A_{1}, 2 A_{3}+A_{1}, 3 A_{2}, A_{3}+2 A_{1}, A_{2}+A_{1} .
$$

For the last four cases, Theorem 2.3 follows from the arguments in Examples 1.61.9 .

Now we consider the remaining case Sing $Y=D_{4}+3 A_{1}$. Then $\tilde{Y}$ contains a $(-1)$-curve $E_{2}$ meeting the three isolated (-2)-curves in $D$ [ibid, Figure 5]. Since $g$ with $\operatorname{ord}(g)=p \geq 5$ stabilizes every component of $D$, this $g$ fixes the three points $D \cap E_{2}$ and hence $E_{2}\left(\cong \mathbf{P}^{1}\right)$ is $g$-fixed. Blowing up a point on $E_{2} \backslash D$, as above, we will reduce to $Y_{1}$ with $\operatorname{Sing} Y_{1}=2 D_{4}$. Now the uniqueness of such $\langle g\rangle \cong \mathbf{Z} /(p)$ in $\operatorname{Aut}(Y)$, modulo equivariant isomorphism, follows from Theorem 2.3: the case $K_{Y}^{2}=1$. This proves Theorem 2.3 .

\section{ACKNOWLEDGEMENT}

The author would like to thank Professor I. Dolgachev for the discussion on generic del Pezzo surfaces, and Professor M. Reid for the discussion on maps between singular del Pezzo surfaces. This work was partially supported by an Academic Research Fund of National University of Singapore.

\section{REFERENCES}

[dF] Tommaso de Fernex, Birational transformations of prime order of the projective plane, Preprint 2001.

[D] M. Demazure, Lecture Notes in Mathematics, 777 (1980), Springer. MR 82d:14021

[DO] I. Dolgachev and D. Ortland, Point sets in projective spaces and theta functions, Astérisque, Vol. 165 (1988). MR 90i:14009

[G] M. H. Gizatullin, Rational G-surfaces, Math. USSR Izv. 16 (1981), 103-134. MR 81d:14020

[GPZ] R. V. Gurjar, C. R. Pradeep, and D.-Q. Zhang, On Gorenstein surfaces isomorphic to $\mathbf{P}^{2} / G$, Nagoya Math. J. to appear, math.AG/0112242.

[H1] T. Hosoh, Automorphism groups of cubic surfaces, J. Algebra 192 (1997), 651-677. MR 99d:14042

[H2] T. Hosoh, Automorphism groups of quartic del Pezzo surfaces, J. Algebra 185 (1996), 374-389. MR 97i:14026

[I] V. A. Iskovskih, Minimal models of rational surfaces over arbitrary fields, Math. USSR Izv. 14 (1981), 17-39. MR 80m:14021

[K] S. Kantor, Theorie der endlichen Gruppen von eindeutigen Transformationen in der Ebene, Berlin: Mayer \& Muller, 1895.

[Ka] Y. Kawamata, A generalization of Kodaira-Ramanujam's vanishing theorem, Math. Ann. 261 (1982), 43-46. MR 84i:14022

[Ko] M. Koitabashi, Automorphism groups of generic rational surfaces, J. Algebra 116 (1988), 130 -142. MR 89f:14045

$[\mathrm{KM}]$ J. Kollar and S. Mori, Birational geometry of algebraic varieties, Cambridge Tracts in Mathematics, 134 (1998). MR 2000b:14018

[M1] Yu. I. Manin, Rational surfaces over perfect fields, II, Math. USSR Sb. 1 (1967), 141-168. MR 37:1374

[M2] Yu. I. Manin, Cubic forms : Algebra, geometry, arithmetic, 2nd ed, North-Holland Math. Library, 4 (1986), North-Holland Publ. Co., Amsterdam-New York. MR 87d:11037

$[\mathrm{MM}]$ M. Miyanishi and K. Masuda, Open algebraic surfaces with finite group actions, Transform. Group, to appear.

[MP] R. Miranda and U. Persson, On extremal rational elliptic surfaces, Math. Z. 193 (1986), 537-558. MR 88a:14044

[MZ1] M. Miyanishi and D.-Q. Zhang, Gorenstein log del Pezzo surfaces of rank one, I; J. Algebra 118 (1988), 63-84; 156 (1993), 183-193. MR 89i:14033 
[MZ2] M. Miyanishi and D.-Q. Zhang, Gorenstein log del Pezzo surfaces of rank one, II; J. Algebra 118 (1988), 63-84; 156 (1993), 183-193. MR 94m:14045

[MZ3] M. Miyanishi and D. -Q. Zhang, Equivariant classification of Gorenstein open log del Pezzo surfaces with finite group actions, Preprint 2001.

[O] K. Oguiso, Automorphism groups in a family of K3 surfaces, math.AG/0104049.

[OS] K. Oguiso and T. Shioda, The Mordell-Weil lattice of a rational elliptic surface, Comment. Math. Univ. St. Paul. 40 (1991), 83-99. MR 92g:14036

[S] B. Segre, The non-singular cubic surfaces, Oxford University Press, Oxford, 1942. MR 4:254b

[V] E. Viehweg, Vanishing theorems, J. Reine Angew. Math. 335 (1982), 1-8. MR 83m:14011

[Y1] Q. Ye, On Gorenstein log del Pezzo surfaces, Japanese J. Math. to appear, math.AG / 0109223.

[Y2] Q. Ye, On algebraic surfaces with non-positive Kodaira dimension, Ph.D. thesis, National Univ. of Singapore, 2001.

[ZD] D.-Q. Zhang, Automorphisms of finite order on rational surfaces, with an appendix by I. Dolgachev, J. Algebra 238 (2001), 560-589. MR 2002c:14064

[Z2] D.-Q. Zhang, Automorphisms of finite order on extremal rational elliptic surfaces and Gorenstein del Pezzo surfaces of degree one, Preprint 2001.

Department of Mathematics, National University of Singapore, 2 Science Drive 2, Singapore 117543, Republic of Singapore

E-mail address: matzdq@math.nus.edu.sg 